\title{
BI-DISSIPATIVE THICK LEVEL SET (TLS) DAMAGE MODEL FOR QUASI-BRITTLE MATERIALS
}

\author{
G. RASTIELLO* ${ }^{*}$ N. MOËS ${ }^{\dagger}$ AND C. COMI ${ }^{\ddagger}$ \\ * École Centrale de Nantes, GeM Institute, UMR CNRS 6183 \\ Nantes, France \\ e-mail: giuseppe.rastiello@ec-nantes.fr \\ $\dagger$ École Centrale de Nantes, GeM Institute, UMR CNRS 6183 \\ Nantes, France \\ e-mail: nicolas.moes@ec-nantes.fr \\ $\ddagger$ Politecnico di Milano, Department of Civil and Environmental Engineering \\ Milano, Italy \\ e-mail: claudia.comi@polimi.it
}

Key words: Bi-dissipatve damage, Quasi-brittle material, Nonlocal damage model, Thick Level Set

\begin{abstract}
The Thick Level Set (TLS) approach applied to damage models allows for a nonlocal treatment that prevents from spurious localization problems. In previous works, isotropic damage models with a single scalar parameter were adopted. Under these conditions, a single level set was used to separate the undamaged zone from the damaged zone, and damage growth was expressed as a level set propagation. Schematically, in the damaged zone, the damage variable directly depended on the level set through an explicit function. Beyond a critical length, material was assumed as fully damaged, thus allowing for a natural transition from damage to localized cracking (i.e., strain localization). In this paper, a first step toward the extension of the TLS approach to "bi-dissipative" isotropic damage models is presented. In the adopted formulation, degradation of material properties under prevailing compressive/tensile loading conditions is separately treated. Two distinct damage variables and activation criteria are used. This allows taking into account the degradation of material properties in tension due to compressive damage (for the moment, no unilateral effect is accounted for). In the article, the local damage model and its mathematical properties are discussed first. A strategy for dealing with bi-dissipative damage in the framework of a TLS approach is then presented.
\end{abstract}

\section{INTRODUCTION}

Continuum damage models are often used to simulate fracture of quasi-brittle materials in reason of their relative simplicity and well established theoretical framework (see e.g. [1]). However, from a mathematical viewpoint, it is well known that when the material softens the mechanical problem exhibits solutions that are not realistic from the physical point of view. From a numerical viewpoint, this leads to a strong dependency on the mesh adopted for the spatial discretization of the computational domain.

Different methods were presented in the literature in order to regularize the damage evolution. One should cite, among others, integral non-local models [2, 3], gradient-enhanced models [4-6], phase-field models [7,8]. Some open questions concerning their capabilities in properly predicting damage initiation and 
growth, up to the formation of localized cracks still exists.

The Tick Level Set (TLS) method [9] provides an alternative theoretical/numerical framework for the regularization of the mechanical problem through the introduction of a characteristic length. Furthermore, by coupling continuum damage mechanics, level setapproaches and strong discontinuity methods (XFEM) the TLS approach provides a natural way for modeling damage growth up to fracture (i.e., cracking) [10-15].

In previous works on the TLS method, simple isotropic damage models with a single scalar parameter were adopted to represent the material response. However, as shown by many authors, more complex damage laws are needed for properly model damage process in cracking concrete and concrete structures. In particular, one would represent at least [16, 17]: 1) different degradation processes under compression and tension; 2) some stiffness recovering associated with crack re-closure (i.e., the so-called unilateral effect).

The formulation of a TLS damage model accounting for these complex mechanical responses is the main finality of the present work.

The paper is structured in four parts as follows. The basic aspects of the TLS method are presented first. A well-posed bi-dissipative continuum damage model for quasi-brittle materials is then presented (no unilateral effect is accounted for in this phase, for sake of simplicity). The term bi-dissipative is used to denote that mechanical responses of the material under prevailing compressive and tensile conditions are separately modeled through two distinct damage variables and activation criteria. This model is then used to develop a TLS damage formulation coupling local and non-local damage evolutions, and allowing to deal with multiple damage variables and activation criteria. In the final part of the paper a completely explicit solution procedure is presented and discussed. Some conclusive remarks close the paper.

\section{THICK LEVEL SET (TLS) METHOD}

The main ingredients of the TLS method are provided in this section. For further details concerning the theoretical basis and numerical formulation, the interested reader can refer to cited works by Moës and co-workers.

\subsection{Main idea}

Consider a solid body $\Omega$ composed by a material which is supposed to behave according to a scalar isotropic damage model with a single damage parameter $d \in[0,1]$. It rises from zero (sound material) to unity (completely damaged material) respecting the following Kuhn-Tucker conditions:

$$
f \leq 0, \quad \dot{d} \geq 0, \quad f \dot{d}=0
$$

where $f$ is a damage activation criterion and $\dot{d}$ denotes the rate of change of the damage parameter.

The main idea of the TLS method applied to quasi-brittle fracture is to impose that the spatial gradient of damage field on $\Omega$ is always bounded [14]:

$$
\|\nabla d\| \leq g(d) \quad \text { on } \quad \Omega
$$

where $g(d)$ is a damage dependent function (it a data function in the model).

The above condition implies that whenever the field $d$ has no sharp gradients (i.e., it is sufficiently smooth) the local model is considered valid, while non-locality comes into play when the inequality is violated. This allows reducing the computational cost related to non-locality: non-locality is activated only in the localization zone (i.e., where $\|\nabla d\|=g(d)$ ) while the damage evolution model remains local in the complementary part of the computational domain (i.e., where $\|\nabla d\|<g(d)$ ).

\subsection{Damage shape function}

In the TLS method the crack is defined as the sub-domain $\Omega_{c} \subset \Omega$ such that $d=1$. Finding numerically this set of points may however reveal quite expensive and tedious. To overcome such a problem, the TLS method expresses the 
damage variable $d$ as a function of a surrogate (upperly unbounded) variable $\phi \in[0,+\infty[$ :

$$
d=d(\phi)
$$

As illustrated in Figure 1, this choice naturally leads to the introduction of characteristic length scale $\ell_{c}$. This allows for defining the crack $\Omega_{c}$ by the set of points:

$$
\Omega_{c}=\left\{\mathbf{x} \in \Omega: \phi \geq \ell_{c}\right\}
$$

The boundary $\Gamma_{c}$ of this zone defines the lips of the crack:

$$
\Gamma_{c}=\left\{\mathbf{x} \in \Omega: \phi=\ell_{c}\right\}
$$

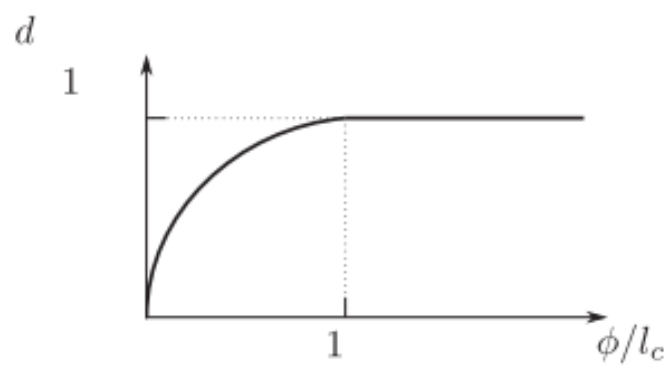

Figure 1: Example of damage shape function.

Function $d(\phi)$ (3), the so-called "damage shape function", is an user-defined input data of the model. It is chosen to be monotonically increasing with respect to $\phi$ and to ensure the following properties:

$$
\left\{\begin{array}{l}
\left.d(\phi) \in \mathcal{C}^{0}\right] 0,+\infty[ \\
d(\phi)=0 \quad \text { if } \phi=0 \\
d(\phi)=1 \quad \text { if } \quad \phi \in\left[\ell_{c},+\infty[\right. \\
\left.d(\phi) \in \mathcal{C}^{1}\right] 0, \ell_{c}[
\end{array}\right.
$$

Using the change of variables (3), the damage rate $\dot{d}$ can be expressed as:

$$
\dot{d}=d^{\prime}(\phi) \dot{\phi}
$$

where $d^{\prime}(\phi)=\mathrm{d} d / \mathrm{d} \phi \geq 0$ is the derivative of $d$ with respect to $\phi$, and $\dot{\phi}$ denotes the rate of change of function $\phi$. As $d^{\prime}(\phi)$ is known and positive, the whole damage model can be now rewritten by making figuring $\dot{\phi}$ instead of $\dot{d}$ (condition $\dot{d} \geq 0$ is now replaced by $\dot{\phi} \geq 0$ ).

\subsection{Coupling local and non-local damage}

Provided that $g(d)=d^{\prime}(\phi)$, condition (2) can be rewritten as $\|\nabla \phi\| \leq 1$. The nonlocality activation condition is thus expressed through the Eikonal equation:

$$
\|\nabla \phi\|=1
$$

while the damage model remains local in the zones where:

$$
\|\nabla \phi\|<1
$$

In the following, we denote $\Omega^{-}$the local zones, while $\Omega^{+}$is for sub-domains where the condition $(8)$ is satisfied (i.e., non-locality is active). Furthermore, we define the so-called damage front (i.e., the evolving interface between the local and nonlocal zones) as:

$$
\Gamma=\bar{\Omega}^{+} \cap \bar{\Omega}^{-}
$$

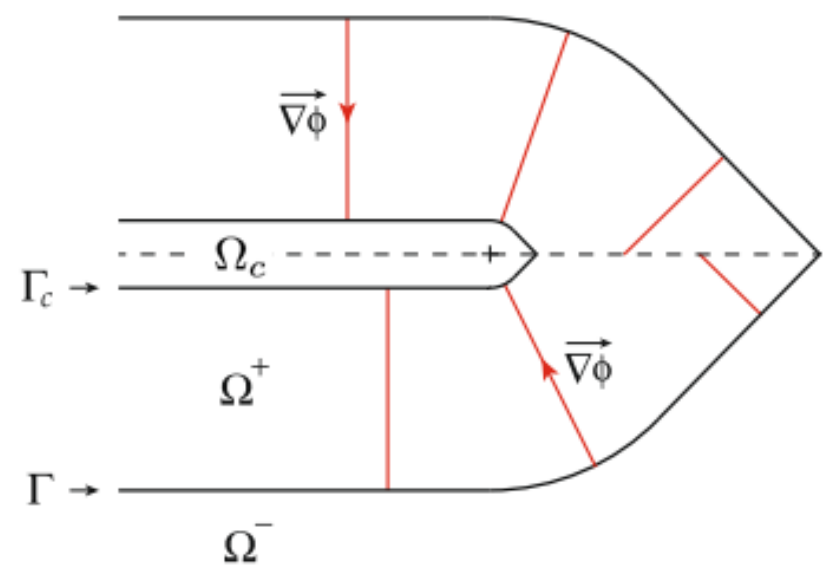

Figure 2: Local $\Omega^{-}$and non-local $\Omega^{+}$domains. $\Gamma_{c}$ denotes the lips of the crack, i.e., the contour of the zone where $d=1$ (or equivalently $\phi \geq \ell_{c}$ ). Note that $\nabla \phi$ is discontinuous along the dashed line (i.e., the skeleton of the distance function)

As a direct consequence of (8), $\phi$ can be seen as a signed Cartesian distance function. Furthermore, to preserve this property, its rate of change $\dot{\phi}$ must satisfy the following condition [18]: $\nabla \dot{\phi} \cdot \nabla \phi=0$. In other words, $\dot{\phi}$ is uniform on each segment aligned with $\nabla \phi$. We denote this space as:

$$
\begin{aligned}
\dot{\phi} & \in \mathcal{A} \\
& =\left\{a \in L^{2}(\Omega): \nabla a \cdot \nabla \phi=0 \text { on } \Omega^{+}\right\}
\end{aligned}
$$




\subsubsection{Non-local damage evolution model}

Once these ingredients are introduced, the non-local damage evolution model is obtained by decomposing $\Omega^{+}$into a set of segments, independent and aligned with $\nabla \phi$, and finding a value $\dot{\phi} \in \mathcal{A}$ over each of them.

The local constitutive model is now expressed in terms of non-local quantities:

$$
\bar{f} \leq 0, \quad \dot{\phi} \geq 0, \quad \bar{f} \dot{\phi}=0
$$

were $\bar{f}$ denotes some averaged value (over each segment) of the damage activation criterion. This is the sole non-local quantity of the model, while remaining quantities are always local.

In variational form, the averaging procedure corresponds to finding $\bar{f} \in \mathcal{A}$ such that:

$$
\begin{aligned}
\int_{\Omega \backslash \Omega_{c}} \bar{f} d^{\prime}(\phi) a \mathrm{~d} \omega & \\
& =\int_{\Omega \backslash \Omega_{c}} f d^{\prime}(\phi) a \mathrm{~d} \omega \quad \forall a \in \mathcal{A}
\end{aligned}
$$

Note that this does not means that $\bar{f}=f$ on the whole domain $\Omega \backslash \Omega_{c}$, because $\bar{f}$ and $f$ on $\Omega^{+}$does not belong to the same functional spaces. To illustrate this aspect, consider a mono-dimensional domain $\Omega$. Under these conditions $\bar{f} \in \mathcal{A}$ and $a \in \mathcal{A}$ are constant over each segment $\left[x_{1}, x_{2}\right] \in \Omega^{+}$, while $f$ is not. Averaging formula (13) thus reads:

$$
\bar{f}=\frac{\int_{x_{1}}^{x_{2}} f d^{\prime}(\phi) \mathrm{d} x}{\int_{x_{1}}^{x_{2}} d^{\prime}(\phi) \mathrm{d} x} \quad \text { (1D case) }
$$

The equality $\bar{f}=f$ is however ensured on $\Omega^{-}$because, due to definition (11), they now belong to the same space. Kuhn-Tucker conditions (12) are thus completely general and valid for the whole domain $\Omega \backslash \Omega_{c}$.

In previous works [9, 11, 12, 15], the TLS method was applied to the regularization of very simple scalar isotropic damage models. A first attempt in extending this theoreti$\mathrm{cal} /$ numerical framework for introducing more complex isotropic damage laws is presented in the following.

\section{BI-DISSIPATIVE DAMAGE MODEL}

A well-posed bi-dissipative damage model is presented briefly. It is mainly based on the formulation originally proposed by [16,17]. Some modifications were however introduced in order to enhance damage activation criteria to ensure the existence and unicity of damage rates. Furthermore (in this phase) the unilateral effect is not modeled for sake of simplicity.

\subsection{Free energy}

With $d_{t} \in[0,1]$ and $d_{c} \in[0,1]$ the damage variables representing material degradation under prevailing actions of tension and compression, the free energy density $\psi=\psi\left(\boldsymbol{\epsilon}, d_{t}, d_{c}\right)$ of the material is written as follows:

$$
\begin{aligned}
\psi=\frac{1}{2}\left(1-d_{t}\right)\left(1-d_{c}\right) & \\
& {\left[2 \mu \mathbf{e}: \mathbf{e}+K(\operatorname{tr} \boldsymbol{\epsilon})^{2}\right] }
\end{aligned}
$$

where $\mu$ and $K$ are the shear and bulk moduli of the sound material, $\epsilon$ is the small strain tensor and e is its deviatoric part. Symbol ":" denotes the contraction operator between fourth-order tensors, while "tr" is the trace operator. The corresponding Cauchy stress tensor $\boldsymbol{\sigma}$ reads:

$$
\begin{aligned}
\boldsymbol{\sigma} & =\partial_{\boldsymbol{\epsilon}} \psi \\
& =\left(1-d_{t}\right)\left(1-d_{c}\right)[2 \mu \mathbf{e}+K \operatorname{tr} \boldsymbol{\epsilon} \mathbf{1}]
\end{aligned}
$$

where symbol $\partial_{\eta}$ denotes the partial derivative operator with respect to the variable $\eta$ and 1 is the second order identity tensor.

According to equations (15)-(16), material stiffness vanishes when $d_{t} \rightarrow 1$ and/or $d_{c} \rightarrow 1$ (i.e., no unilateral effect associated with crack re-closure can be modeled). Note, that it could be reintroduced easily into (15), e.g. by splitting the volumetric deformation into its positive and negative parts, and assuming that the free energy under hydrostatic compression depends on $d_{c}$ only [16].

\subsection{Damage activation criteria}

Dissipative processes under prevailing states of tension and compression are treated separately by introducing two distinct damage activation criteria $\left(f_{t}, f_{c}\right)$. Evolutions of the scalar 
damage variables $\left(d_{t}, d_{c}\right)$ satisfy Kuhn-Tucker conditions:

$$
\begin{aligned}
& f_{t} \leq 0, \quad \dot{d}_{t} \geq 0, \quad f_{t} \dot{d}_{t}=0 \\
& f_{c} \leq 0, \quad \dot{d}_{c} \geq 0, \quad f_{c} \dot{d}_{c}=0
\end{aligned}
$$

\subsubsection{Damage criteria in stress space}

Damage activation criteria are defined in the stress space as follows:

$$
\begin{aligned}
& f_{t}\left(\boldsymbol{\sigma}, d_{c}, d_{t}\right)=J_{2}(\boldsymbol{\sigma})-a_{t} I_{1}^{2}(\boldsymbol{\sigma}) \\
& \quad+b_{t} I_{1}(\boldsymbol{\sigma}) m_{t}\left(d_{t}, d_{c}\right)-k_{t} m_{t}^{2}\left(d_{t}, d_{c}\right)
\end{aligned}
$$

and

$$
\begin{aligned}
f_{c}\left(\boldsymbol{\sigma}, d_{c}\right) & =J_{2}(\boldsymbol{\sigma})+a_{c} I_{1}^{2}(\boldsymbol{\sigma}) \\
& +b_{c} I_{1}(\boldsymbol{\sigma}) m_{c}\left(d_{c}\right)-k_{c} m_{c}^{2}\left(d_{c}\right)
\end{aligned}
$$

where $J_{2}(\boldsymbol{\sigma})$ denotes the second invariant of the stress tensor, $I_{1}(\boldsymbol{\sigma})$ is its first invariant, and $a_{t}, b_{t}, k_{t}, a_{c}, b_{c}, k_{c}$ are non-negative material parameters. Finally, functions $m_{t}\left(d_{t}, d_{c}\right)$ and $m_{c}\left(d_{c}\right)$ are:

$$
\begin{aligned}
m_{t}\left(d_{t}, d_{c}\right) & =\left(1-d_{c}\right) r_{t}\left(d_{t}\right) \\
m_{c}\left(d_{c}\right) & =r_{c}\left(d_{c}\right)
\end{aligned}
$$

where $r_{t}$ and $r_{c}$ are isotropic hardeningsoftening functions. They are computed according to the following relationship (for $i=t, c$ ):

$$
\begin{aligned}
& r_{i}\left(d_{i}\right)= \\
& \begin{cases}1-\left(1-\frac{\sigma_{e i}}{\sigma_{0 i}}\right)\left(1-\frac{d_{i}}{d_{0 i}}\right)^{2}, & d_{i}<d_{o i} \\
{\left[1-\left(\frac{d_{i}-d_{0 i}}{1-d_{0 i}}\right)^{c_{i}}\right]^{\beta_{i}},} & d_{i} \geq d_{o i}\end{cases}
\end{aligned}
$$

where $\sigma_{e i} / \sigma_{0 i}$ denotes the ratio of the stress at elastic limit to the stress at peak in an uniaxial tension/compression test, $d_{0 i}$ is the damage level corresponding to peak-stress, $c_{i}$ is a scalar parameter controlling the slope of softening branch of $r_{i}\left(d_{i}\right)$, and $\beta_{i}$ is a material parameter (assumed equal to 3/4). For more details on representative values of material parameters introduced above, one can refer to [16].

The intersections of surfaces $f_{t}\left(\boldsymbol{\sigma}, d_{c}, d_{t}\right)=$ 0 and $f_{c}\left(\boldsymbol{\sigma}, d_{c}\right)=0$ with the plane $\left(I_{1}, \sqrt{J}_{2}\right)$ are respectively a hyperbola and an ellipse, both symmetric with respect to the horizontal axis $I_{1}$. A schematic representation of the initial linear elastic domain, and its evolution with damage is provided in Fig. 3 .
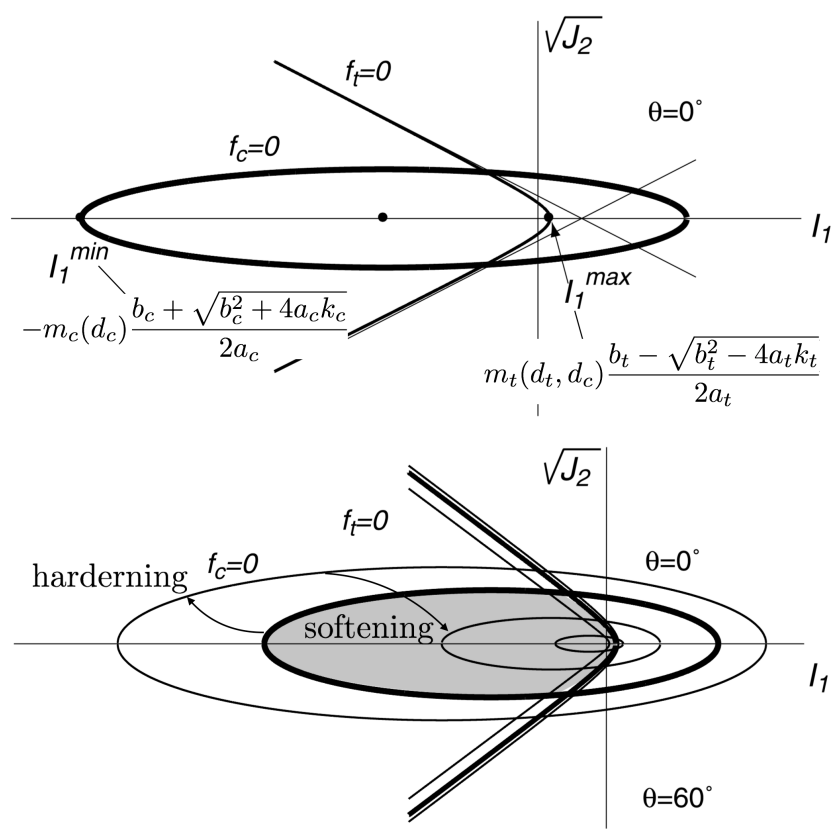

Figure 3: Schematic representation of the linear elastic domain and its evolution with damage

Finally, note that according to equations (19)-(20) tensile damage activation criterion is influenced by compressive damage while not vice-versa.

\subsubsection{Damage criteria in strain space}

Criteria (19) and (20) are rewritten in strain space as follows:

$$
\begin{aligned}
f_{t}^{*}\left(\boldsymbol{\epsilon}, d_{t}\right) & =J_{2}(\boldsymbol{\epsilon})-a_{t}^{*} I_{1}^{2}(\boldsymbol{\epsilon}) \\
& +b_{t}^{*} I_{1}(\boldsymbol{\epsilon}) m_{t}^{*}\left(d_{t}\right)-k_{t}^{*} m_{t}^{* 2}\left(d_{t}\right)
\end{aligned}
$$

and

$$
\begin{aligned}
& f_{c}^{*}\left(\boldsymbol{\epsilon}, d_{t}, d_{c}\right)=J_{2}(\boldsymbol{\epsilon})+a_{c}^{*} I_{1}^{2}(\boldsymbol{\epsilon}) \\
& \quad+b_{c}^{*} I_{1}(\boldsymbol{\epsilon}) m_{c}^{*}\left(d_{c}, d_{t}\right)-k_{c}^{*} m_{c}^{* 2}\left(d_{c}, d_{t}\right)
\end{aligned}
$$


where (for $i=c, t)$ :

$$
\begin{aligned}
f_{i}^{*}\left(\boldsymbol{\epsilon}, d_{t}, d_{c}\right) & =\frac{f_{i}\left(\boldsymbol{\sigma}, d_{c}, d_{t}\right)}{4 \mu^{2}\left(1-d_{t}\right)^{2}\left(1-d_{c}\right)^{2}} \\
a_{i}^{*} & =\frac{9 K^{2}}{4 \mu^{2}} a_{i} \\
b_{i}^{*} & =\frac{3 K}{4 \mu^{2}} b_{i} \\
k_{i}^{*} & =\frac{1}{4 \mu^{2}} k_{i} \\
m_{t}^{*}\left(d_{t}\right) & =\frac{r_{t}\left(d_{t}\right)}{\left(1-d_{t}\right)} \\
m_{c}^{*}\left(d_{t}, d_{c}\right) & =\frac{r_{c}\left(d_{c}\right)}{\left(1-d_{t}\right)\left(1-d_{c}\right)}
\end{aligned}
$$

These criteria will be used in next sections to formulate the TLS damage model.

\subsection{Existence/unicity of damage rates}

Damage activation functions (24) and (25) are slightly different from those proposed by [16]. Minor enhancements were introduced in order to ensure a priori the existence and unicity of tensile and compressive damage rates for a given strain rate.

To prove this property, consider the problem starting from a point belonging to both of the damage activation surfaces. When both criteria are active, one can write:

$$
\begin{array}{lll}
f_{t}^{*} \leq 0 & \dot{d}_{t}^{*} \geq 0 & \dot{f}_{t}^{*} \dot{d}_{t}=0 \\
f_{c}^{*} \leq 0 & \dot{d}_{c}^{*} \geq 0 & \dot{f}_{c}^{*} \dot{d}_{c}=0
\end{array}
$$

or, equivalently, in matrix form:

$$
\left\{\begin{array}{l}
\mathbf{z}=\mathbf{H} \dot{\mathbf{d}}+\mathbf{b}(\dot{\boldsymbol{\epsilon}}) \geq 0 \\
\dot{\mathbf{d}} \geq 0 \\
\mathbf{z} \cdot \dot{\mathbf{d}}=0
\end{array}\right.
$$

where matrix $\mathbf{H}$ and the unknown solution vector $\dot{\mathrm{d}}$ are:

$$
\begin{aligned}
\mathbf{H} & =\left[\begin{array}{cc}
h_{t t} & 0 \\
h_{c t} & h_{c c}
\end{array}\right]=\left[\begin{array}{cc}
-\partial_{d_{t}} f_{t}^{*} & 0 \\
-\partial_{d_{t}} f_{c}^{*} & -\partial_{d_{c}} f_{c}^{*}
\end{array}\right], \\
\dot{\mathbf{d}} & =\left[\dot{d}_{t}, \dot{d}_{c}\right]^{T}
\end{aligned}
$$

Provided the form of matrix $\mathbf{H}$, a solution $\dot{\mathrm{d}}$ of the Linear Complementary Problem (LCP) (35) exists and is unique (for any $\dot{\boldsymbol{\epsilon}}$ ) if matrix $\mathbf{H}$ is definite positive. For the considered model, this condition can be easily proved after some simple algebraic passages. In other words:

$$
h_{t t}>0 \quad h_{c c}>0
$$

for any admissible set of model/material parameters [16]. Positiveness of damage rates also ensures the positiveness of the total dissipation $\mathcal{D}$.

\section{TLS BI-DISSIPATIVE DAMAGE MODEL}

Due to a separate treatment of degradation processes under tension and compression, the constitutive model presented in previous section provides a good starting point for developing TLS damage formulations accounting for multidissipative processes. This section provides the main aspects of the TLS model proposed to deal with two damage variables (and evolution criteria) using a single level-set function.

\subsection{Equivalent damage variable}

According to standard TLS arguments (see Sec. 2), the damage variable $d$ is computed as a function of a surrogate function $\phi$. Using two distinct damage variables $\left(d_{t}, d_{c}\right)$ should therefore require the use of two distinct levelset functions. Although this could appear to be a quite natural choice, it may reveal complex in terms of theoretical formulation and numerical implementation. That's why, in this paper, we propose to control the evolution of two damage variables through a single level-set function $\phi$.

For this purpose, we introduce an "equivalent damage variable" $d \in[0,1]$ which is supposed to evolve as a function of $\phi$ :

$$
1-d(\phi)=\left(1-d_{t}\right)\left(1-d_{c}\right)
$$

Note that similar splits are used to formulate continuum damage models for composite materials and laminates.

This choice allows for strongly simplifying model formulation, but has an important consequence: a unique characteristic length $\ell_{c}$ can 
be introduced in the model through the damageshape function $d(\phi)$. For quasi-brittle materials (and in particular for concrete) this could represent a strong limitation, as different authors evidenced that the characteristic length in compression is larger than those in tension (up to 10 times larger). Some modifications might therefore be needed for enhancing the model on this point.

\subsection{Non-local damage evolution}

According to (39) the TLS framework presented in Sec. 2 2 remains still valid. Some considerations concerning the way for dealing with multiple damage rates and criteria using a single function $\phi$ are however needed. Indeed, for each segment aligned with $\nabla \phi$, the equivalent damage rate $\dot{d}$ is proportional to the rate of change of the level-set function:

$$
\dot{d}=d^{\prime}(\phi) \dot{\phi}=\left(1-d_{c}\right) \dot{d}_{t}+\left(1-d_{t}\right) \dot{d}_{c}
$$

but no information concerning $\left(\dot{d}_{c}, \dot{d}_{t}\right)$ are available.

As a first attempt to overcome this problem, we propose to write tensile and compressive damage rates as follows:

$$
\begin{aligned}
& \dot{d}_{t}=\frac{\alpha}{1-d_{c}} \dot{d}=\frac{d^{\prime}(\phi)}{1-d_{c}} \alpha \dot{\phi} \\
& \dot{d}_{c}=\frac{1-\alpha}{1-d_{t}} \dot{d}=\frac{d^{\prime}(\phi)}{1-d_{t}}(1-\alpha) \dot{\phi}
\end{aligned}
$$

where $\alpha$ ranges between 0 and 1 and is supposed uniform over the segment considered (i.e., $\alpha \in \mathcal{A}$ ). In particular, $\alpha$ is null (equal to unity) when only compressive (tensile) damage occurs, and is in between these extreme values when both activation criteria are satisfied at the same time:

$$
\left\{\begin{array}{lll}
\alpha=1 & \dot{d}_{t}>0 & \dot{d}_{c}=0 \\
\alpha=0 & \dot{d}_{t}=0 & \dot{d}_{c}>0 \\
\alpha \in] 0,1[ & \dot{d}_{t}>0 & \dot{d}_{c}>0
\end{array}\right.
$$

Using decomposition (41)-(42) allows for rewriting the constitutive model in non-local form as follows:

$$
\begin{array}{lll}
\bar{f}_{t}^{*} \leq 0 & \dot{\phi}_{t} \geq 0 & \bar{f}_{t}^{*} \dot{\phi}_{t}=0 \\
\bar{f}_{c}^{*} \leq 0 & \dot{\phi}_{c} \geq 0 & \bar{f}_{c}^{*} \dot{\phi}_{c}=0
\end{array}
$$

where:

$$
\dot{\phi}_{t}=\alpha \dot{\phi} \quad \dot{\phi}_{c}=(1-\alpha) \dot{\phi}
$$

and $\bar{f}_{t}^{*}$ and $\bar{f}_{c}^{*}$ denote the averaged damage activation criteria on segments aligned with $\nabla \phi$.

The constitutive model is thus reformulated in terms $\dot{\phi} \in \mathcal{A}$ and $\alpha \in \mathcal{A}$. As for the monodissipative damage model, Kuhn-Tucker conditions (44)-(45) are valid on both $\Omega^{+}$and $\Omega^{-}$.

\subsection{Boundary value problem}

Once the non-local damage model is written, one can define the boundary value problem. For this purpose, consider a solid body occupying a domain $\Omega$. Its external boundary $\partial \Omega$ comprises two non-overlapping parts $\partial \Omega_{u}$ and $\partial \Omega_{t}$. They are such that displacements $\mathbf{u}=\lambda \mathbf{u}^{0}$ are imposed on $\partial \Omega_{u}$ and loadings $\mathbf{t}=\lambda \mathbf{t}^{0}$ are applied on $\partial \Omega_{t}$. Parameter $\lambda$ is a loading factor and upscript " 0 " denotes some reference value.

Under quasi-static conditions in the absence of body forces, solving the boundary value problem corresponds to finding an admissible displacement field ${ }^{1}$.

$$
\begin{array}{r}
\mathbf{u} \in \mathcal{U}=\left\{\mathbf{u}^{*}\right. \text { regular enough : } \\
\int_{\Omega \backslash \Omega_{c}} \psi(\boldsymbol{\epsilon}(\mathbf{u}), d(\phi)) \mathrm{d} \omega<+\infty \\
\left.\mathbf{u}^{*}=\lambda \mathbf{u}^{0} \text { on } \partial \Omega_{u}\right\}
\end{array}
$$

and an admissible level-set function:

$$
\begin{aligned}
& \phi \in \Phi=\left\{\phi^{*} \in \mathcal{C}^{0}(\Omega):\right. \\
&\left\|\nabla \phi^{*}\right\|=1 \text { on } \Omega^{+} \\
&\left.\left\|\nabla \phi^{*}\right\|<1 \text { on } \Omega^{-}\right\}
\end{aligned}
$$

\footnotetext{
${ }^{1}$ As in [14], we request that the energy is finite. This space is not simply $H^{1}$, as in elasticity, since the stiffness is allowed to vanish on $\Gamma_{c}$.
} 
such that the following conditions are satisfied as a whole:

$$
\left\{\begin{array}{lll}
\mathbf{u} \in \mathcal{U}, \phi \in \Phi & & \\
\operatorname{div} \boldsymbol{\sigma}=0 & \text { on } & \Omega \backslash \Omega_{c} \\
\boldsymbol{\sigma}=\partial_{\boldsymbol{\epsilon}} \psi & \text { on } & \Omega \backslash \Omega_{c} \\
\boldsymbol{\epsilon}=\operatorname{Sym}(\nabla \mathbf{u}) & \text { on } & \Omega \backslash \Omega_{c} \\
\mathbf{u}=\lambda \mathbf{u}^{0} & \text { on } & \partial \Omega_{u} \\
\boldsymbol{\sigma} \cdot \mathbf{n}=\lambda \mathbf{t}^{0} & \text { on } & \partial \Omega_{t} \\
\boldsymbol{\sigma} \cdot \mathbf{n}=0 & \text { on } & \Gamma_{c} \\
\text { Eqs. 44) }-45) & \text { on } & \Omega \backslash \Omega_{c}
\end{array}\right.
$$

In previous equations, symbol "Sym" denotes the symmetric part operator. Furthermore, note that the crack is considered stress-free (secondlast condition).

\section{CONCLUSIVE REMARKS}

This paper presented some elements of a novel TLS damage formulation allowing for the implementation of complex constitutive equations accounting for multi-dissipative degradation mechanisms.

A well-posed bi-dissipative continuum damage model for quasi-brittle materials was presented first. Mechanical responses of the material under prevailing compressive/tensile conditions were separately modeled through two distinct damage variables and activation criteria.

This model was then used to develop a TLS formulation coupling local and non-local damage evolutions. It allows for dealing with multiple damage variables and activation criteria though a single level set function. The boundary value problem was finally presented.

Numerical computations are actually in progress.

\section{REFERENCES}

[1] Lemaitre, J., and Desmorat, R. (2005). Continuum Damage Mechanics. Springer Berlin Heidelberg.

[2] Pijaudier, C. G., and Bazant, Z. P. (1987). Non local Damage Theory. Asce, 113(10), 15121533.
[3] Bažant, Z. P., and Jirásek, M. (2002). Nonlocal Integral Formulations of Plasticity and Damage: Survey of Progress. Journal of Engineering Mechanics, 128(11), 11191149. http://doi.org/10.1061/(ASCE)07339399(2002)128:11(1119)

[4] Frémond, M., and Nedjar, B. (1996). Damage, gradient of damage and principle of virtual power. International Journal of Solids and Structures, 33(8), 10831103.

[5] Comi, C. (1999). Computational modelling of gradient-enhanced damage in quasi-brittle materials. Mechanics of Cohesive-Frictional Materials, 36(April 1997), 1736.

[6] Peerlings, R. H. J., Geers, M. G. D., de Borst, R., and Brekelmans, W. A. M. (2001). A critical comparison of nonlocal and gradient-enhanced softening continua. International Journal of Solids and Structures, 38(44-45), 77237746.

[7] Amor, H., Marigo, J.-J., and Maurini, C. (2009). Regularized formulation of the variational brittle fracture with unilateral contact: Numerical experiments. Journal of the Mechanics and Physics of Solids, 57(8), 12091229. http://doi.org/10.1016/j.jmps.2009.04.011

[8] Miehe, C., Welschinger, F., and Hofacker, M. (2010). Thermodynamically consistent phase-field models of fracture: Variational principles and multi-field FE implementations. International Journal for Numerical Methods in Engineering, 83(10), 12731311. http://doi.org/10.1002/nme.2861

[9] Moës, N., Stolz, C., Bernard, P.-E., and Chevaugeon, N. (2011). A level set based model for damage growth: The thick level set approach. International Journal for $\mathrm{Nu}-$ merical Methods in Engineering, 86(3), 358380 . 
[10] Stolz, C., and Moës, N. (2012). A new model of damage: a moving thick layer approach. International Journal of Fracture, 174(1), 4960.

[11] Bernard, P. E., Moës, N., and Chevaugeon, N. (2012). Damage growth modeling using the Thick Level Set (TLS) approach: Efficient discretization for quasistatic loadings. Computer Methods in Applied Mechanics and Engineering, 233236,1127

[12] Cazes, F., and Moës, N. (2015). Comparison of a phase-field model and of a thick level set model for brittle and quasi-brittle fracture. International Journal for Numerical Methods in Engineering, 103(2), 114143.

[13] Parrilla Gómez, A., Moës, N., and Stolz, C. (2015). Comparison between thick level set (TLS) and cohesive zone models. Advanced Modeling and Simulation in Engineering Sciences, 2(1), 18. http://doi.org/10.1186/s40323-015-00419

[14] Moës, N., Stolz, C., and Chevaugeon, N. (2014). Coupling local and non-local dam- age evolutions with the Thick Level Set model. Advanced Modeling and Simulation in Engineering Sciences, 1(1), 16.

[15] Moreau, K., Moës, N., Picart, D., and Stainier, L. (2015). Explicit dynamics with a non-local damage model using the thick level set approach. International Journal for Numerical Methods in Engineering, 102(3-4), 808838.

[16] Comi, C., and Perego, U. (2001). Fracture energy based bi-dissipative damage model for concrete. International Journal of Solids and Structures, 38(36-37), 64276454

[17] Comi, C. (2001). Non-local model with tension and compression damage mechanisms. European Journal of Mechanics, A/Solids, 20(1), 122.

[18] Sethian, J.A., Level Set Methods and Fast Marching Methods: Evolving Interfaces in Computational Geometry, Fluid Mechanics, Computer Vision and Materials Science.Cambridge University Press, 1999 